Research Paper

\title{
Yes-Associated Protein (Yap) Is Necessary for Ciliogenesis and Morphogenesis during Pronephros Development in Zebrafish (Danio Rerio)
}

\author{
Liangliang $\mathrm{He}^{1}$, Wenyan $\mathrm{Xu}^{2}$, Ying Jing ${ }^{1}$, Ming $\mathrm{Wu}^{1}$, Shuwei Song${ }^{1}$, Ying $\mathrm{Cao}^{2 \varpi}$, Changlin $\mathrm{Mei}^{1 凶}$ \\ 1. Kidney Institute of CPLA, Division of Nephrology, Changzheng Hospital, Second Military Medical University, NO.415 Fengyang Road, \\ Shanghai 200003, China. \\ 2. School of life science and technology, Tongji University ,1239 Siping Road, Shanghai 200092, China \\ $\triangle$ Corresponding authors: yingcao@tongji.edu.cn (Cao Y); chlmei1954@126.com (Mei CL) \\ (C) 2015 Ivyspring International Publisher. Reproduction is permitted for personal, noncommercial use, provided that the article is in whole, unmodified, and properly cited. \\ See http://ivyspring.com/terms for terms and conditions.
}

Received: 2014.12.14; Accepted: 2015.04.23; Published: 2015.06.11

\begin{abstract}
The Hippo signaling pathway and its transcriptional co-activator Yap are known as essential regulators for cell proliferation and organ size. However, little is known about their roles in kidney development and ciliogenesis. We examined expression of Yap during zebrafish embryogenesis, and its transcripts were detected in pronephric duct, while Yap protein was found to be localized in the cytoplasm and apical membrane in kidney epithelium cells. By morpholino (MO) knockdown of yap expression in zebrafish, the injected larve exhibits pronephic cysts and many aspects of ciliopathy, which can be rescued by full-length yap mRNA, but not yap ${ }^{5127 \mathrm{~A}} \mathrm{mRNA}$. With transgenic $\mathrm{Tg}\left(\mathrm{Na}^{+} / \mathrm{K}^{+}\right.$ATPase:EGFP $)$, we found that lacking Yap led to expansion and discontinuities of pronephric duct, as well as disorganization of cloaca during pronephros morphogenesis. Mis-located $\mathrm{Na}^{+} / \mathrm{K}^{+}$ATPase and ciliary abnormalities are also detected in pronephric duct of yap morphants. In addition, genetic analysis suggests that yap interacts with ift20, ift88 and arl $3 \mathrm{~b}$ in pronephric cyst formation. Taken together, our data reveals that Yap is required for pronephric duct integrity, maintenance of baso-lateral cell polarity, and ciliogenesis during zebrafish kidney development.
\end{abstract}

Key words: Yap; Pronephros; Cyst; Kidney; Cilia; Zebrafish

\section{Introduction}

Cilia are microtubule-based organelles extending outward from the basal body and ubiquitously exist in almost all vertebrates. Cilia function in fluid flow generation and integration of environmental cues, playing a critical role in vertebrate physiology and development. Recent years, cilia begin to receive extensive attention since it has been linked to a number of phenotypes, such as hydrocephalus, lateral defects, polycystic liver and kidney disease, retinal degeneration. Mutations in ciliary genes lead to a broad set of developmental and adult human diseases including nephronophthisis, Bardet-Biedl syndrome and Joubert syndrome[1-3]. Genetic and proteome screens have revealed an expanding list of proteins required for generation and maintenance of cilia[4].
Polycystic kidney disease (PKD) is a group of genetic diseases featuring the growth of numerous kidney cysts, which is one of the most common potentially lethal genetic disorders in human[5]. More and more evidences have shown that structural or functional ciliary defects cause PKD. Pathogenic proteins of human autosomal dominant PKD (Polycystin-1 and Polycystin-2) have been detected in renal cilium[6,7]. The best studied ciliary trafficking protein complexes named intraflagellar transport (IFT) particles, which are organized into distinct A/B complexes, are responsible for retrograde or anterograde transport of the moving cargo between the cell body and the axonemal distal tip respectively. IFT mutant mouse and zebrafish have been reported to develop 
kidney cyst[8-13]. Other examples as small GTPase Arl13b, transition zone complexes and BBSome have also showed associations with ciliogenesis and PKD[14-19].

The Hippo pathway is a highly conserved signaling regulating cell growth, differentiation, embryogenesis and tumorigenesis[20-26]. The core pathway in mammalian is that Mst kinase in complex with scaffold protein Sav1, phosphorylates downstream kinase Lats, which in turn phosphorylates transcriptional co-activators Yap and Taz, leading to their cytoplasmic retention. When the Hippo pathway is inactive, dephosphorylated Yap/Taz accumulates in nucleus and promotes transcription of the target genes[27]. Upstream of the core pathway lies a number of cell surface regulators, including cadherins, cell polarity complexes, and GPCRs[28-30].

Previous studies have reported disrupted Hippo pathway in PKD and ciliogenesis. TAZ knockout mice exhibit renal cysts, fewer and shorter cilia in cystic lining epithelia, with down-regulation of ciliary genes as Kif3a, Phkd1[31-33]. Knockdown of Yap in zebrafish leads to pronephric cyst formation[29]. More recently, Yap nuclear localization is observed in cystic epithelia and dilated tubules of PKD mouse models and patients, suggesting its essential role in progression of PKD[34]. However, the regulating process is barely known.

The zebrafish has emerged as a vertebrate animal model exceptionally suitable for the study of embryogenesis and cyst formation. In this study, we analyzed zebrafish yap mRNA expression and protein sublocalization in pronephric duct during embryogenesis. Using antisense morpholino to block the translation of zebrafish Yap, the embryos phenocopy many aspects of ciliopathy and develop pronephric cyst. Detections of these embryos with live, in vivo cell imaging, in situ hybridization and immunostaining provide evidence that defective pronephros development and cyst formation in yap knockdown embryos are correlated with disrupted cell migration, altered basolateral cell polarity and ciliary abnormalities. Further, genetic analysis shows Yap interacts with IFT B complex particles and Joubert syndrome protein Arl13b in cyst formation, suggesting an intricate relationship between Yap and ciliogenesis.

\section{Results}

\section{Expression and subcelluar localization of Zebrafish Yap in Developing Embryos}

During zebrafish embryogenesis, yap has been shown as a maternal gene, and the zygotic expression starting from the mid-blastula transition stage was mainly in notochord, eyes, brain, which were almost cilia related organs[20].The zebrafish pronephric ducts are also composed of ciliated cells, so we performed in situ hybridization (ISH) and observed that yap was modestly expressed in the lateral plate mesoderm (LPM) and intermediate mesoderm (IM) from the six-somite stage, and was subsequently appeared in the developing pronephric ducts until at least 3 days post fertilization (d.p.f) (Fig.1A-E), suggesting its potential role in the pronephros development.

Previous study of mammalian YAP demonstrated its dynamical expression during kidney organgenesis[21]. To examine the subcellular Yap distribution in zebrafish pronephro, we implemented whole-mount immunostaining and its expression was detected in the pronephric duct at 24 and 48 hours post fertilization (h.p.f) (Fig.1F-H). The specificity of YAP antibody was demonstrated by western blotting of 1 d.p.f embryos with overexpression of Yap-EGFP or knockdown of endogenous Yap with an antisense MO (Fig.1I). We also injected one-cell zebrafish embryos with plasmid pTol2-8kb-EGFP-yap and Tol2 transposase mRNA to drive fusion protein EGFP-YAP to pronephric duct, and detected a comformity of the subcellular localization of exogenetic and endogenous Yap. Magnification view of both anterior and posterior segment of a single pronephric duct revealed that Yap diffusely localized in the cytoplasm ringing the nucleus with a concentration near the apical surface. We also double stained the injected embryos with anti-atypical PKC (aPKC) to highlight the apical surface and found that it was co-localized with Yap (Fig.1J-J').

\section{Phenotypic changes after knockdown of zebrafish yap}

To determine the role of Yap in zebrafish pronephros development, we used antisense MO against the $5^{\prime}$-UTR to effect a targeted knockdown of Yap. The $\mathrm{MO}$ has been previously shown to be efficient for abrogation of gene function in the zebrafish[20] and its effectiveness was confirmed by western blot as well (Fig.1I). Compared with the injection control, yap morphants revealed multiple defects from 2.5 d.p.f including pronephric cyst, pericardial edema, hydrocephalus, smaller eyes, a short curved tail, and defective cloaca (Fig.2A-E and Supplementary Fig.S1), which were consistent with yap expression pattern shown by ISH. The phenotypes of yap morphants were dose dependent and initial experiments were conducted to identify $1.5 \mathrm{pmol}$ as the most effective dose for the MO (Supplementary Fig.S1C and D). Since yap morphants showed apoptosis in head during embryogenesis, we co-injected with 0.5 pmol $t P 53 \mathrm{MO}$ and excluded the possibility that these phenotypes were nonspecific effects due to up-regulation 
of the p53 apoptosis pathway[35] (Supplementary Fig.S1B and C). More importantly, Yap knockdown induced phenotypes can be rescued with zebrafish full-length yap mRNA, demonstrating specificity of the MO. Further, we decided to make clear the functional significant of Yap's activity. The S127 and transcription activation domain is well conserved in zebrafish Yap, which influence the activity form and target genes transcription respectively. We found yap ${ }^{\text {S127A }}$ mutant mRNA cannot rescue kidney cyst in yap morphants while overexpressing $\triangle \mathrm{TAD}$ Yap without the transcription activation domain is sufficient to rescue the phenotype (Fig.2I). With Tol2 Kit [36], we also discerned that the fluorescence of mutant S127A Yap was translocated into nucleus with remaining stain near the apical surface (Fig.2F-F'). These results suggest that phosphorylated but not activated Yap played a more important role in zebrafish pronephro development, and maintaining normal subcellular localization may be necessary for Yap's function.
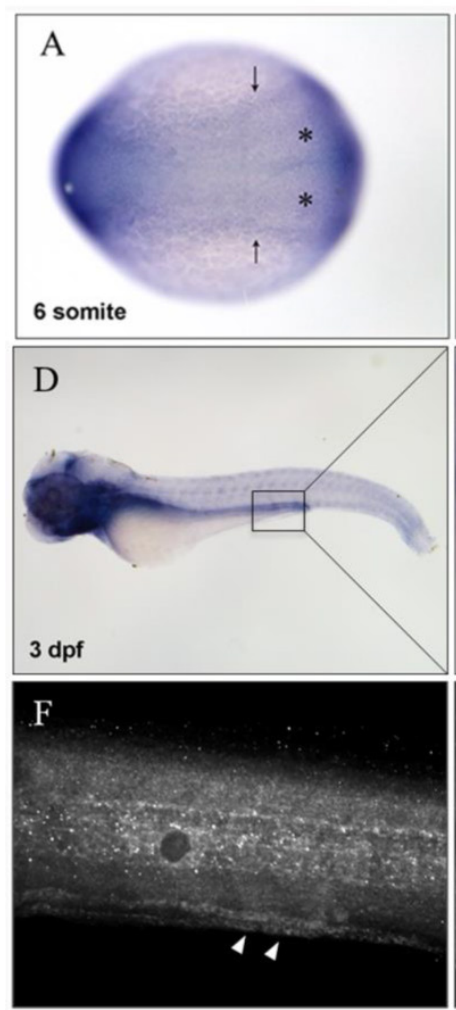

I

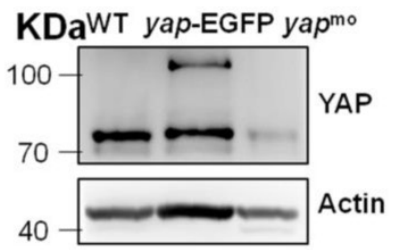

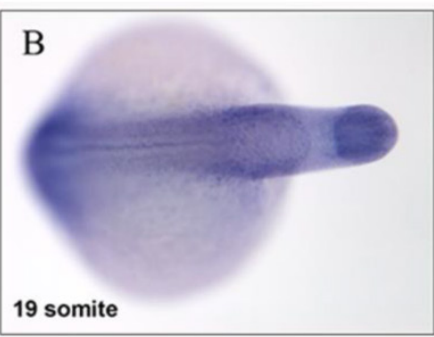
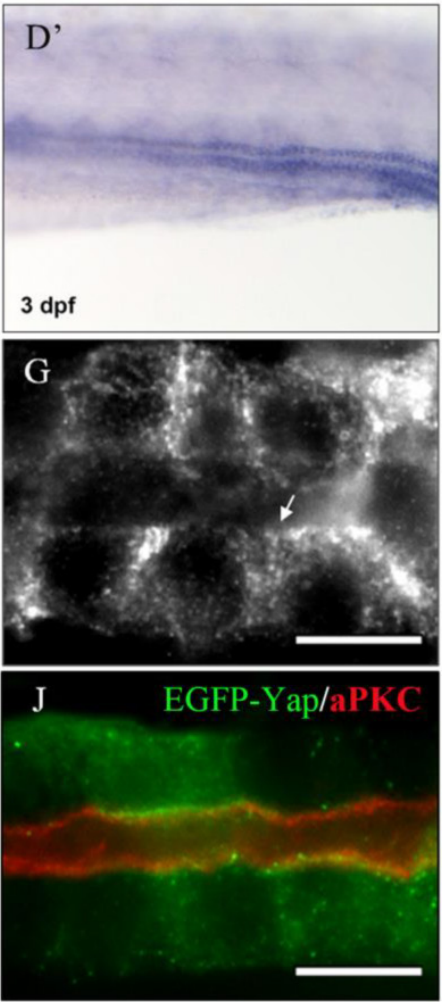
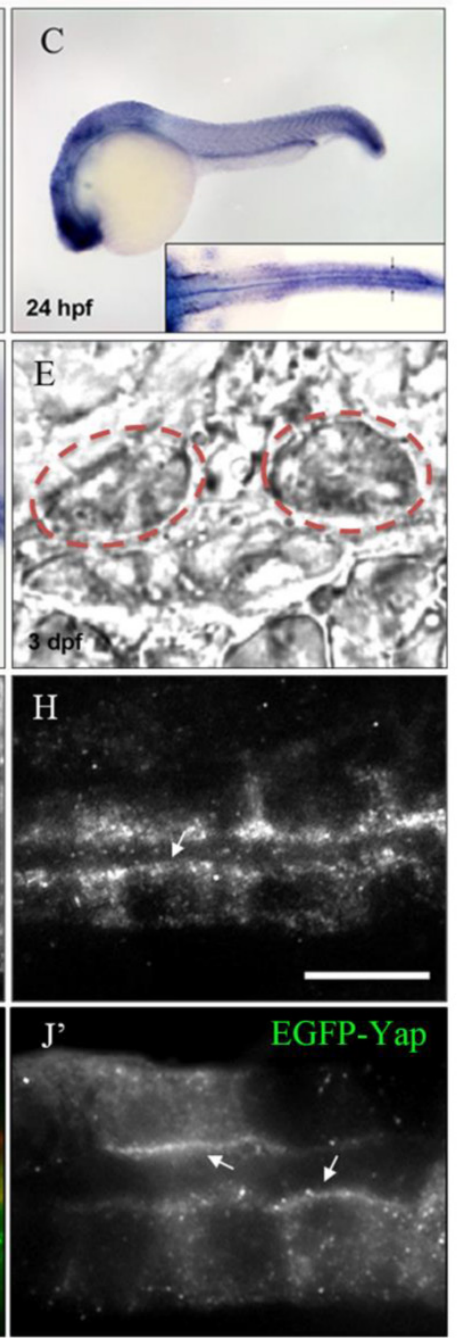

Figure 1. yap expression and protein sublocalization during zebrafish nephrogenesis. (A) By whole-mount in situ hybridization, yap is detcted in the LPM (arrows) and IM (asterisk) as early as the 6-somite stage. (B-E) At the 19-somite stage, 24 h.p.f, and 3 d.p.f, yap persists in the pronephric ducts, which is confirmed by dorsal view(C; inset), higher magnification view ( $\left.D^{\prime}\right)$ and cross section view (E; dashed red circles). (F) Expression of Yap protein in the trunk of a 2 d.p.f embryo viewed in whole-mount is modest in the pronephric duct (arrowheads). (G-H) A close view of the anterior $(\mathrm{G})$ and posterior $(\mathrm{H})$ segment shows that Yap immunofluorescence is distributed throughout the cytoplasm with a concentration near the apical surface (arrows). (I) Western blot of 1 d.p.f embryos with Yap antibody shows the endogenous Yap at approximately 70 KD, and exogenetic Yap-EGFP at 100 KD, while expression of Yap protein in 1pmol yap MO embryos almost disappear. (J-J') With Tol2 kit, exogenetic EGFP-Yap distributes in the cytoplasm and has co-localization with the apical marker aPKC (arrows). Bar: $10 \mu \mathrm{m}$. 


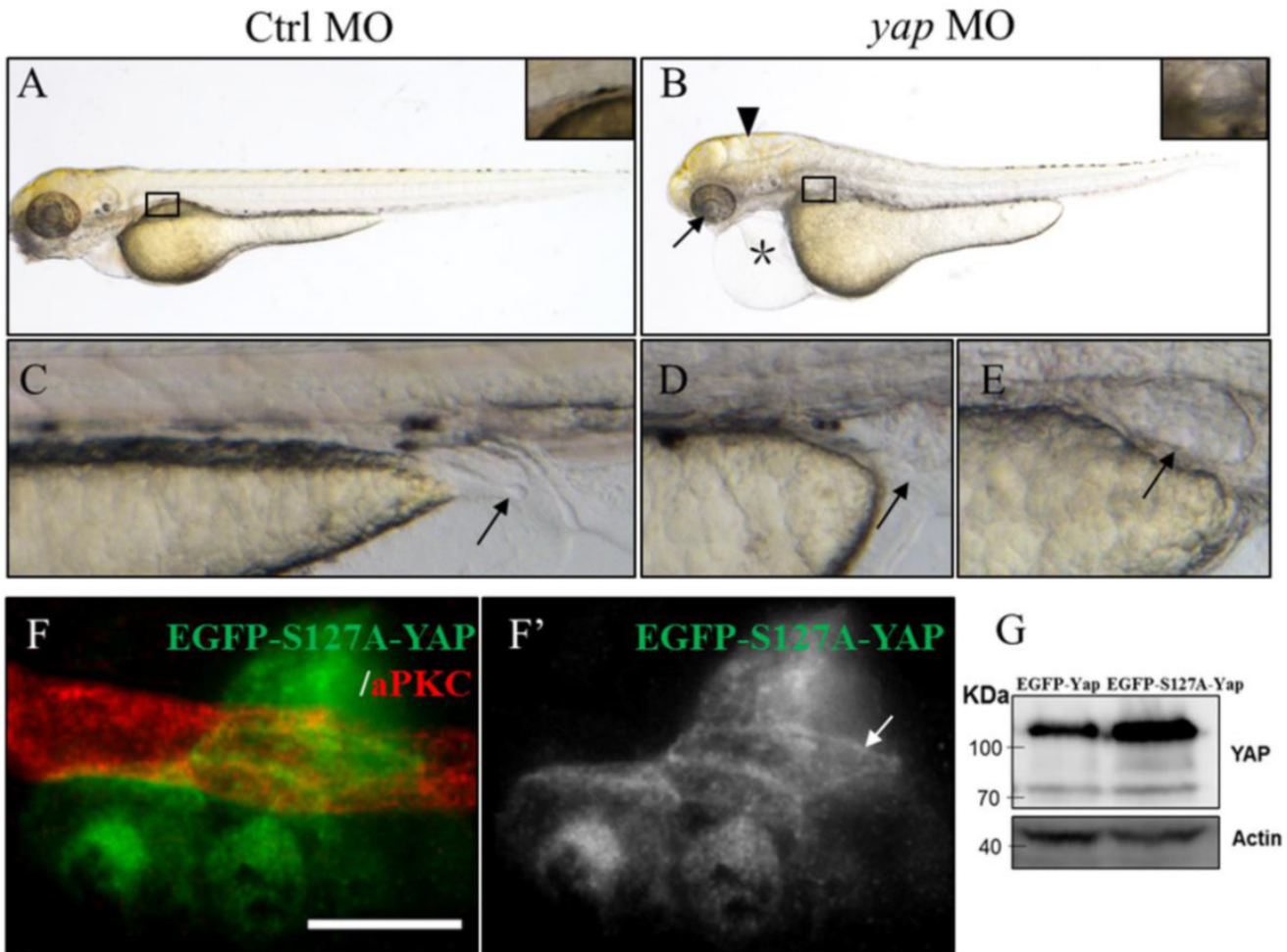

$\mathrm{H}$

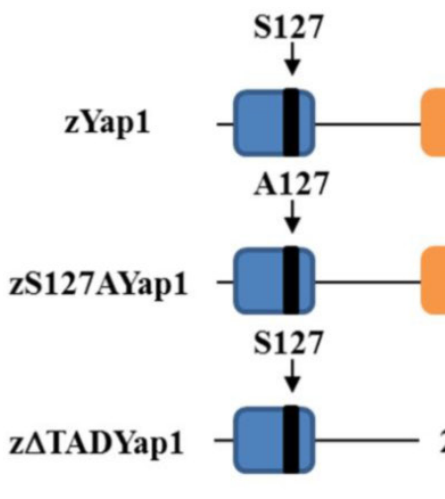

TEAD binding domain

Transcription activation domain
I

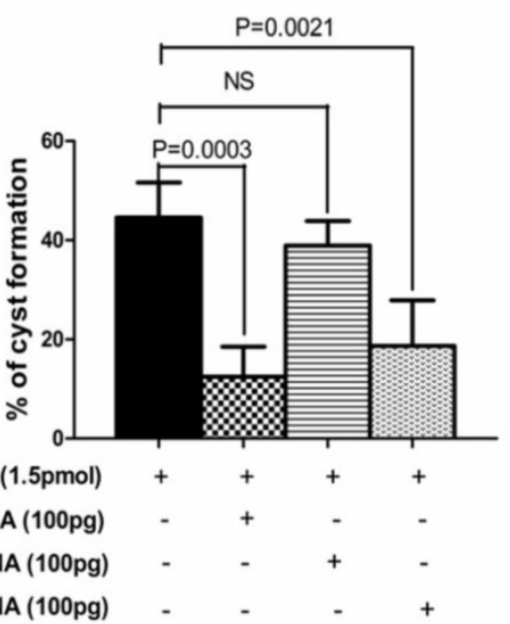

Figure 2. yap knockdown by antisense $M O$ results in abnormal phenotypes which can be rescued by full-length and $\triangle T A D$ yap mRNA. (A-E) Phenotypes of control embryo and yap morphant at 3 d.p.f. (B) yap morphant exhibit glomerular cyst (inset), heart edema (asterisk), hydrocephalus (arrowhead), smaller eyes (arrow), a short and slightly curved tail. In addition, at the distal end of pronephric duct, the control embryo form obvious outlet at cloaca (C; arrow) while in the morphant, the outlet can't be seen (D; arrow) or is obstructed, promoting dilation or cysts formation (E; arrow). (F-F') Immunostaining shows that exogenetic dominant active form EGFP-S127AYap is translocated into nucleus with the apical staining left (F'; arrow). Bar: $10 \mu \mathrm{m}$. (G) Western blot of 2 d.p.f embryos illustrates the protein level of kidney-specifically overexpressed EGFP-Yap and EGFP-S127AYap. (H) Modular structures of zYap1, S127A Yap and $\triangle T A D$ Yap. In zYap, the conserved S127 in the TEAD binding domain (aa7-103) is actually located in the aa87. $\triangle T A D$ mRNA encodes Yap without the transcription activation domain (aa238-442). aa: amino acids. (I) The pronephric cyst of yap MO embryos can be rescued by co-injecting full-length yap mRNA and $\triangle T A D$ yap mRNA $(P<0.01)$, however, co-injecting with yap ${ }^{5 / 27 A}$ mutant mRNA develops cyst at an average of $38.9 \%$, not significantly different from cyst formation of yap morphants with an average of $44.3 \%$.

With $\mathrm{Tg}\left(\mathrm{Na}^{+} / \mathrm{K}^{+}\right.$ATPase:EGFP) fish line, development of pronephric duct is visible, during which the distal tubule cells proliferate and migrate forward, manifesting position and morphology of each nephron segment[37]. We further found loss of integrity of pronephric duct in yap morphants. From 1 d.p.f, distal tubular dilation was seen, and disruption of the posterior segment at cloaca existed, including lack of fusion and cyst formation (Fig.3B; arrow). At 2 d.p.f, we surprisingly found that EGFP labeled pronephric duct was discontinuous (Fig.3D and F; arrowheads), and the ratio increased greatly at 3 d.p.f (Fig.3I), which was mostly detected in the medial tubules. ISH with pronephric duct probe cadherin (cldh) 17 in 3 d.p.f morphants verified the same "gap" pattern (Fig.3H). Besides, blocked cell migration was observed during pronephro development (Fig.3J). 

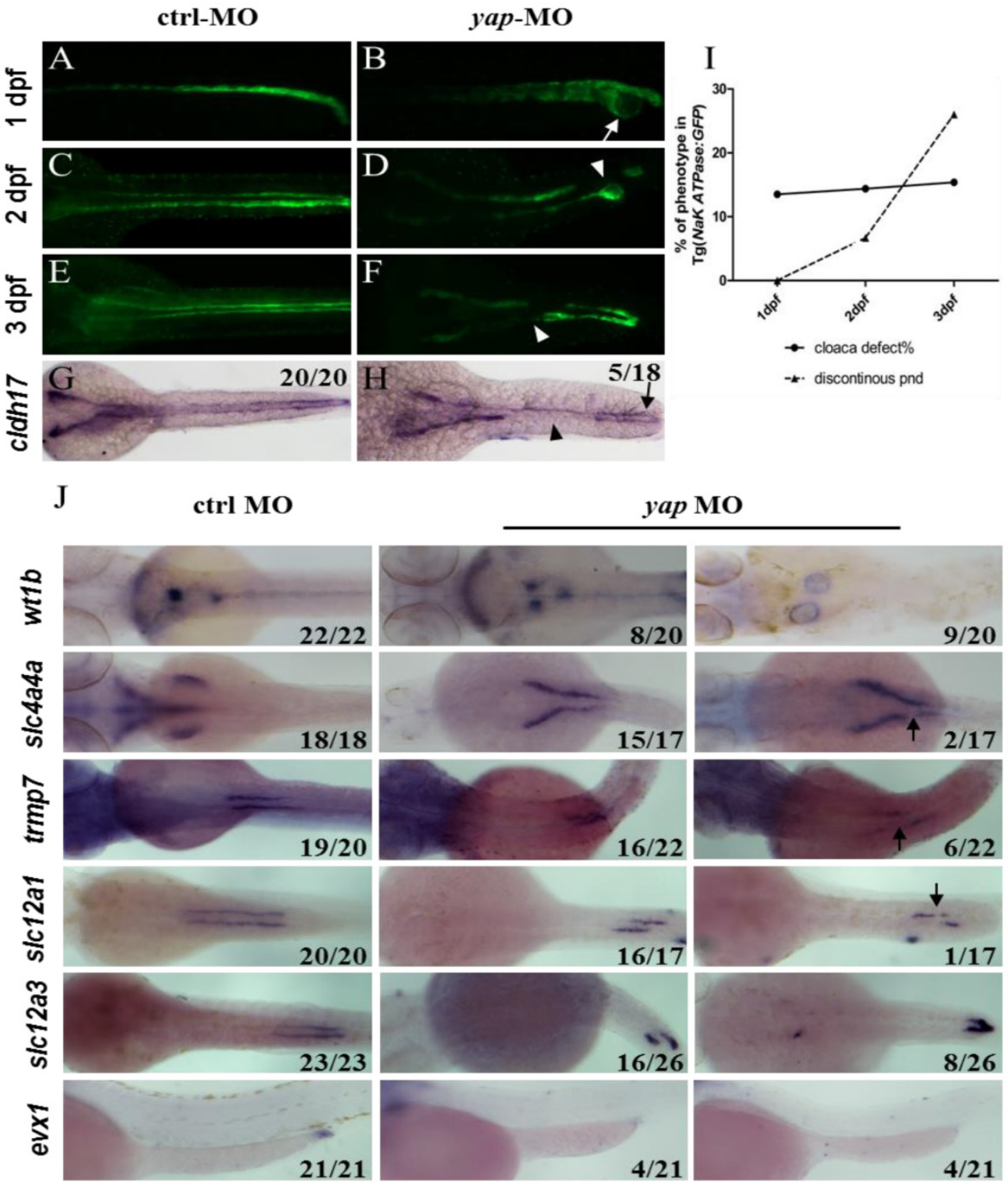

yap MO
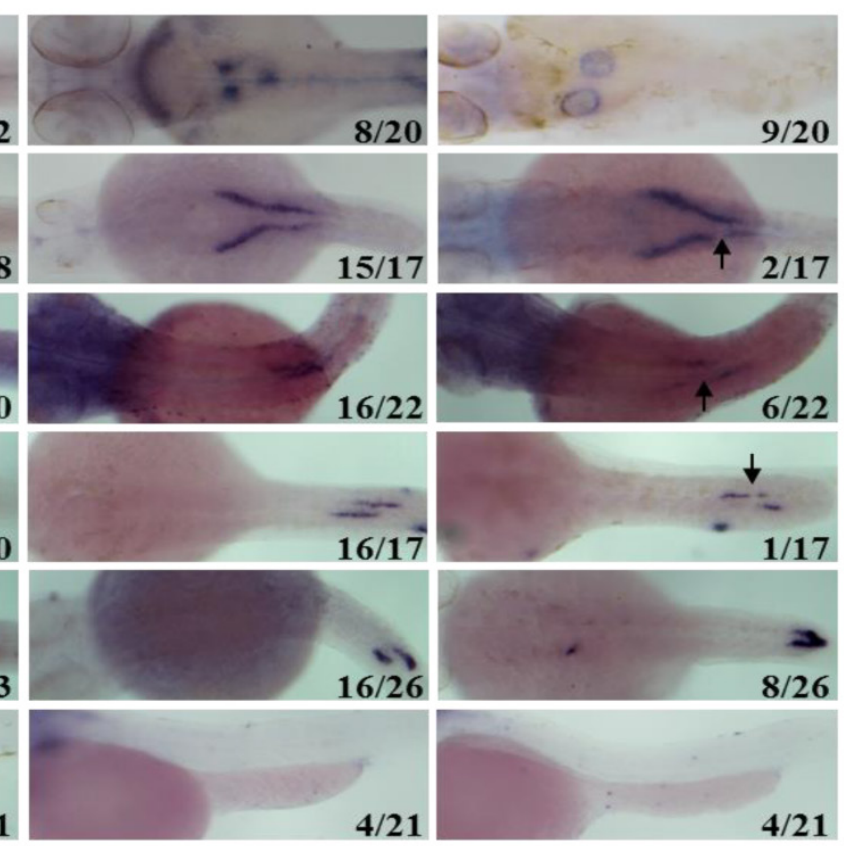

Figure 3. Abberant morphology of pronephric duct develops during collective cell migration. (A-I) Morphology of pronephric duct labeled by $T g\left(N a^{+} / K^{+} A T P a s e: E G F P\right)$ from 1 d.p.f to 3 d.p.f. Disorganization of cloaca occurs in about $15 \%$ embryos from 1 d.p.f (B; arrow). Discontinuous pronephric duct is found from 2 d.p.f in $6.7 \%$ embryos, and the ratio of occurrence increases sharply to $26 \%$ at 3 d.p.f (D and F; arrowheads), cldh 17 probe shows the similar phenotype (H; arrow and arrowhead). (J) ISH of segment-restricted probe at 3 d.p.f. Pronephric cysts are displayed by wt lb. slc $4 a 4 a^{+}$cells fail to migrate towards the glomeruli-neck region compared with the control embryos. Discontinuous segments are found in slc4a4a $a^{+}$trpm- $7^{+}$, slcl $2 a l^{+}$cells (arrowheads). Decreased or disappeared expression of evxl is seen. The numbers on the lower left of each figure represent the counted phenotypic and total embryos. All photos are dorsal views except for evxl, which are lateral views.

PCNA staining showed no change in distal segment cells that excluded the problem with cell proliferation (Supplementary Fig.S3). We next examined a range of segment-restricted markers at 24 h.p.f to assess the pronephro segmentation. The expression pattern of these markers appeared relatively unaffected, demonstrating that the occurrence of defective cloaca and discontinuous pronephric duct was not related with cell fate deficiency (Supplementary Fig.S3). At 3 d.p.f, however, pronephric cysts were displayed by wt1b (glomerular marker; Fig.3J). The distribution of epithelium cells in all segments failed to extend towards the head kidney compared with the control embryos, and proximal segment convolution vanished in slc4a4a+ cells (PCT segment; Fig.3J). We also found discontinuous expression of slc4a4a, trpm-7 (PST segment), slc12a1 (DE segment), and decreased or disappeared expression of evx1 (cloaca maker; Fig.3J). These findings indicate that the defects in pronephric duct develop during collective cell migration and morphogenesis.

\section{Disrupted location of $\mathrm{Na}^{+} / \mathrm{K}^{+}$ATPase in yap morphants}

In yap morphants, cystic dilation in the glomeruli-neck region were first detected under light micros- 
copy at about 50-55 h.p.f. Pronephric cyst is sometimes accompanied by tubular dilation, so we further examined pronephric duct with immunostaining. aPKC indicates the apical membrane as inner diameter of the pronephric duct, and a6F subunit of $\mathrm{Na}^{+} / \mathrm{K}^{+}$ ATPase represents basolateral surface as outer diameter. We found tubular dilation develop in both proximal and distal segments of pronephros in 3 d.p.f morphants, and the proximal tubule remained straight with no sign of "hairpin" convolution (Fig.4A-B and S2A). Some studies have reported that altered apico-basal cell polarity in renal epithelia may lead to tubular expansion and cyst formation, e.g. mis-sorting or insertion of transporters and receptors as $\mathrm{Na}^{+} / \mathrm{K}^{+}$ATPase, NKCC1 and EGFR[38]. Thus we analyzed $\mathrm{Na}^{+} / \mathrm{K}^{+}$ATPase localization in 3 d.p.f embryos with cross section staining. The baso-lateral staining of $\mathrm{Na}^{+} / \mathrm{K}^{+}$ATPase was not obvious in a part of enlarged tubules (Fig.4D'; red arrow), and even apical expression was detected (Fig.4D'; arrowhead). Mis-localization of $\mathrm{Na}^{+} / \mathrm{K}^{+}$ATPase in renal cells can result in secretion of sodium into the lumen and fluid accumulation[39].Meanwhile, location of the apical marker, such as aPKC and F-actin, appeared intact (Fig. 4D and S2B). The results suggest that disruption of baso-lateral cell polarity, but not apical polarity, may give rise to cyst formation in yap morphants.

\section{Ciliary defects in yap morphants}

Defects in ciliary genes often lead to pronephric cyst, curved tails, heart edema, smaller eyes in zebrafish as yap morphants exhibit. To examine the effect of Yap in ciliogenesis, we stained the eyes and pronephric ducts of yap morphants and control embryos with acetylated a-tublin. In zebrafish, the outer segment of the photoreceptor is a highly modified cilium giving access to visual transduction, it begins to form by 54 h.p.f and persists thereafter. We found that the outer segments were almost discernable in smaller eyes of 3 d.p.f yap morphants (Fig.5A; arrows). The pronephric duct features multiciliated cells (MCCs) and single ciliated cells (SCCs), which form clustered cilia bundles and single cilia respectively. The single cilia are observed as early as 20 h.p.f while the bundling cilia can only be detected from about 36 h.p.f. We examined cilia formation at 3 d.p.f in at least 7 embryos each group, and found that the MCCs in the mid region of the duct did not form normal bundles but only disordered cilia, which had also been seen in other zebrafish cilia mutants $[9,39]$, while in the posterior segments, the number $(20.3 \pm 1.5$ in morphant versus $30.3 \pm 2.7$ in wild-type embryos, mean \pm SEM; $P<0.01)$ and length $(3.9 \pm 0.2 \mu \mathrm{m}$ in morphant versus $6.2 \pm 0.2 \mu \mathrm{m}$ in wild-type embryos, mean \pm SEM; $P<0.001)$ of single cilia decreased significantly (Fig.5B-C). More importantly, cilia defects in yap morphants could be rescued by overexpression of full-length yap mRNA or $\triangle T A D$ yap mRNA, but not by yap ${ }^{\text {S127A }}$ mRNA (Supplementary Fig.S4).
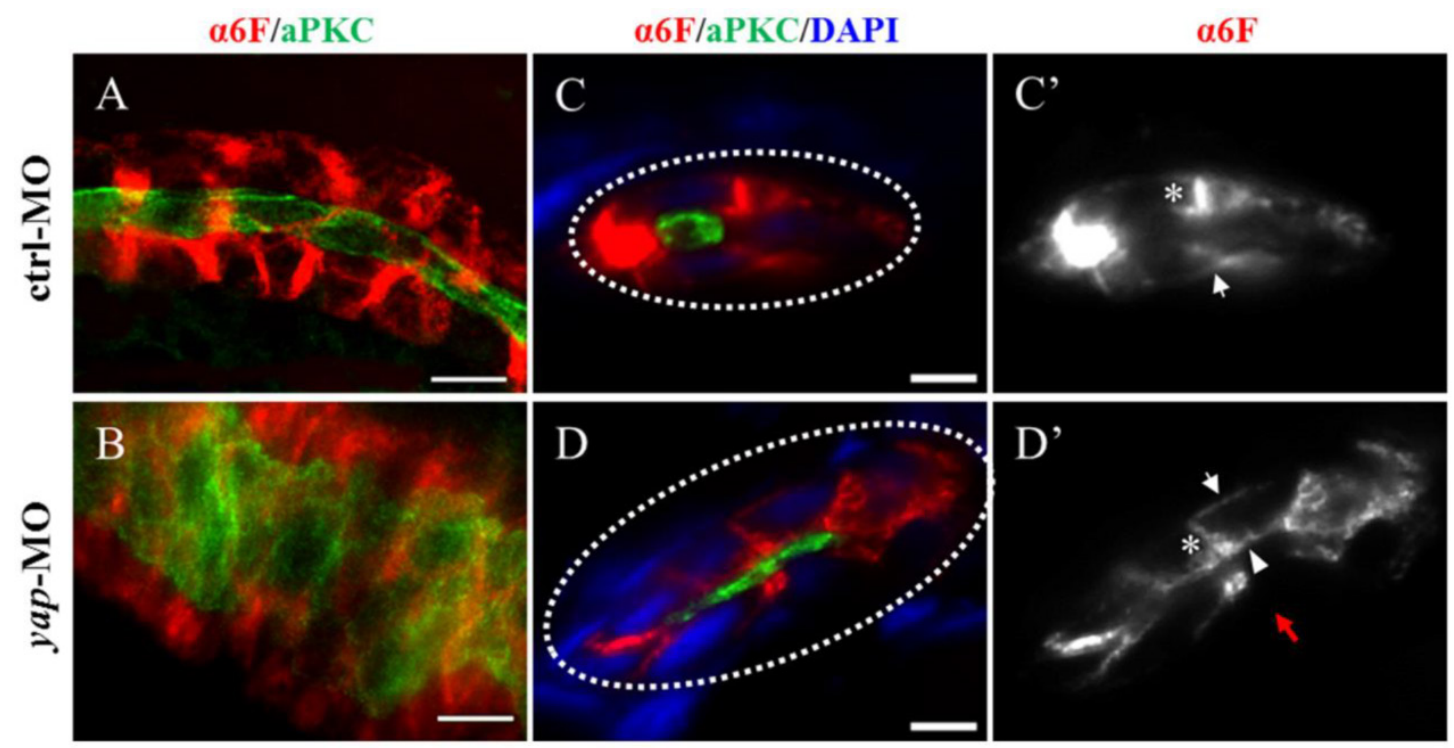

Figure 4. The $\mathrm{Na}^{+} / \mathrm{K}^{+}$ATPase is mis-located to the apical surface of pronephric duct in yap morphants. (A-B) Whole-mount staining shows that the inner diameter of the enlarged duct indicated by aPKC is about fourfold of the control, however, the change of the outer diameter indicated with $\alpha-6 \mathrm{~F}$ is not that dramatic. (C-D') The cross section view demonstrates increased cell number surrounding the dilated duct compared with the control duct (white dashed circles represent the cross sections of the ducts), the baso-lateral staining of $\mathrm{Na}^{+} / \mathrm{K}^{+}$ATPase is not obvious in a part of enlarged tubules (Fig.4D'; red arrow), and even is mis-targeted to the apical surface adjacent to aPKC ( $\mathrm{D}$; arrowhead). (C' and D') Arrows indicate basal surface and asterisks indicate lateral surface. (A-B) Bar: $10 \mu \mathrm{m}$. (C-D) Bar: $5 \mu \mathrm{m}$. 

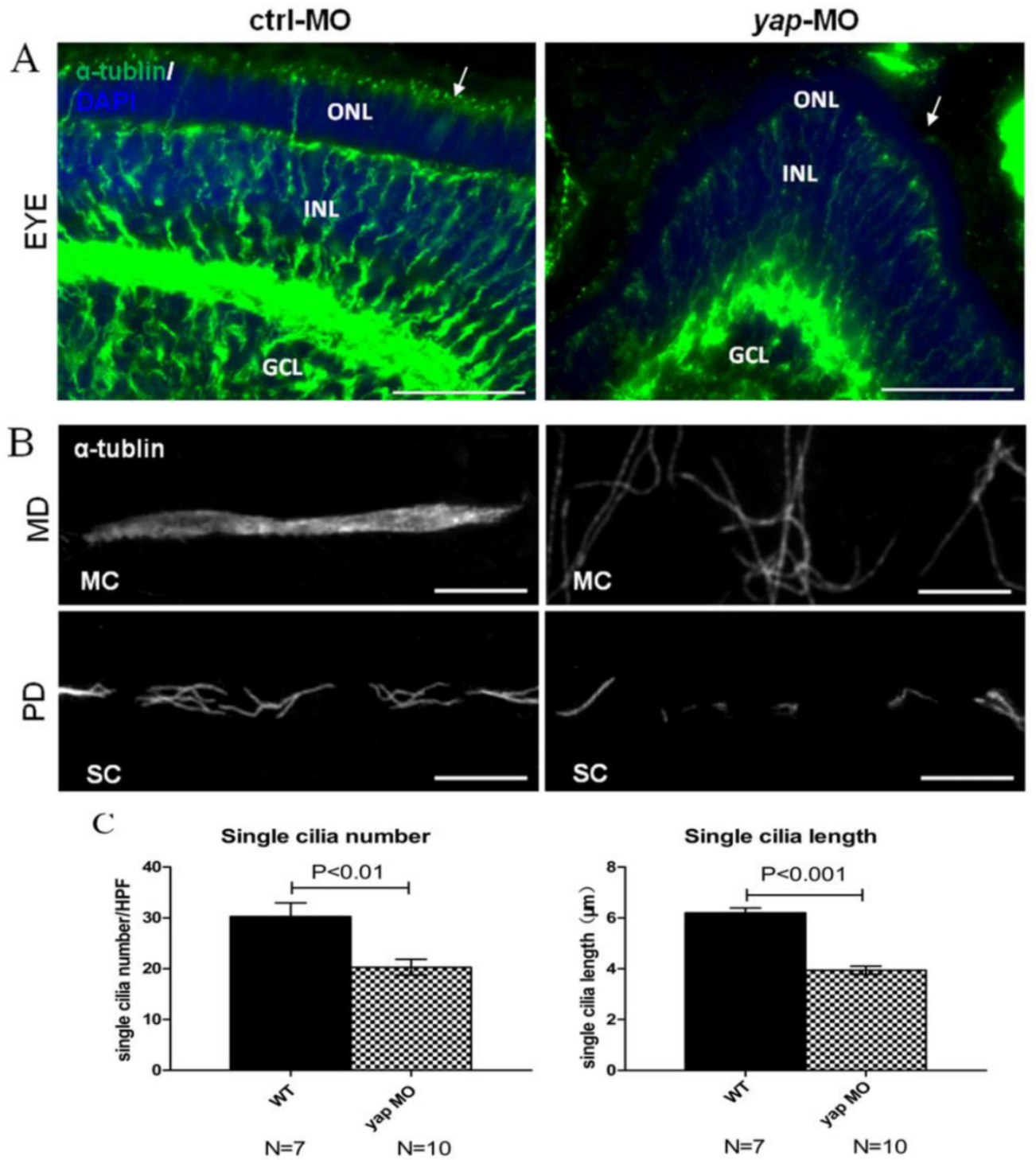

Figure 5. yap morphants have ciliary defects. (A) In the eye, the outer segment of photoreceptor cell, which is a modified cilium, is hard to detect in the morphants at 3 d.p.f (the left arrow indicates the control, the right arrow shows defect in yap morphant). Bar: $25 \mu \mathrm{m}$ (B) Compared with the injection control, cilia defects are observed in medial and posterior segment of the pronephric duct in the morphant. Bar: $10 \mu \mathrm{m}$. (C) Statistical analysis of the single cilia number per high power field and single cilia length in control and morphant embryos. The cilia are shown by staining with antibody against acetylated $\alpha$-tublin. $\alpha$-tub, anti-acetylated $\alpha$-tublin; GCL, ganglion cell layer; INL, inner nuclear layer; $\mathrm{ONL}$, outer nuclear layer; MD, medial segment of pronephric duct; PD, posterior segment f pronephric duct; MC, multicilia; SC, single cilia; HPF, high power field.

\section{Yap influences basal body arrangement and apical membrane docking in ciliogenesis}

To further investigate the ciliary defects of yap morphants, we performed ISHs for foxj1a and shippo1 (odf3b) (Fig.6A). foxj1a is a cilia master regulator and one target of Hedgehog signaling, promoting ciliary differentiation and function, and its activity is sufficient for ectopic cilia development[40]. If the ciliary defects in yap morphants are foxj1a dependent, we would expect decreased level of foxj1a. Actually, we found up-regulation of foxj1a in both 24 h.p.f and 3 d.p.f yap morphants $(90 \%$ and $70.6 \%$ respectively; Fig.6A). We additionally over-expressed foxj1a mRNA in wild-type and yap morphant embryos to observe its influence in pronephro ciliogenesis, However, little change had been seen (Supplementary Fig.S4). The results suggest cilia defects in yap morphants are foxj1 $a$ independent, and enhance of foxj1a may be a feedback regulation induced by epithelial stretch and cyst formation[41]. shippo1 is a marker of MCCs[42].The expression pattern of shippo1 in 24 h.p.f morphants appeared relatively unaffected (86.7\%). But in 3 d.p.f yap morphants, shippo1 labeled MCCs were in a disorganized array and gathered together in the middle region (57.9\%), while in control, MCCs placed one by one along the pronephric duct (Fig.6A). Accumulated MCCs could be attributed to defects in lateral inhibition during differentiation of bipotential precursor cells[42] or blocked collective cell migration. Unchanged shippo $1^{+}$and $\operatorname{trpm} 7^{+}$cells at 24 h.p.f excluded the possibility of biased differentiation to- 
wards a MCC fate (Supplementary Fig.S3). Obviously delayed cell migration of yap morphants at 3 d.p.f could explain gathered MCCs in the medial segment (Fig.3J).

All cilia are extended from basal bodies which are analogous to mitotic centrioles. In both MCCs and SCCs, ciliogenesis begins with basal bodies apical membrane docking and then acquires accessary structure and elongates[43]. By immunostaining with anti- $\gamma$-tublin and anti-aPKC, we additionally found disordered basal bodies in dilated pronephric duct. In whole-mount staining of $3 \mathrm{dpf}$ embryos, basal bodies of a MCC organized in a thread-like array at the apical side in control (Fig.6B'; arrowheads), while in expanded ducts of morphants, the basal bodies of a MCC were accumulated into clusters (Fig.6C'; arrowheads), which had been reported to affect cilia bundling[9]. With cross section staining, failure of basal bodies docking to the apical surface was seen in enlarged pronephric ducts of both anterior and posterior segments, and this might account for cilia defects in SCCs (Fig.6E and G; arrows).

\section{yap genetically interacts with ift20, ift88 and arll3b}

Given yap morphant embryos display shorted cilia length with defective basal body apical docking, we proposed the hypothesis that Yap protein might
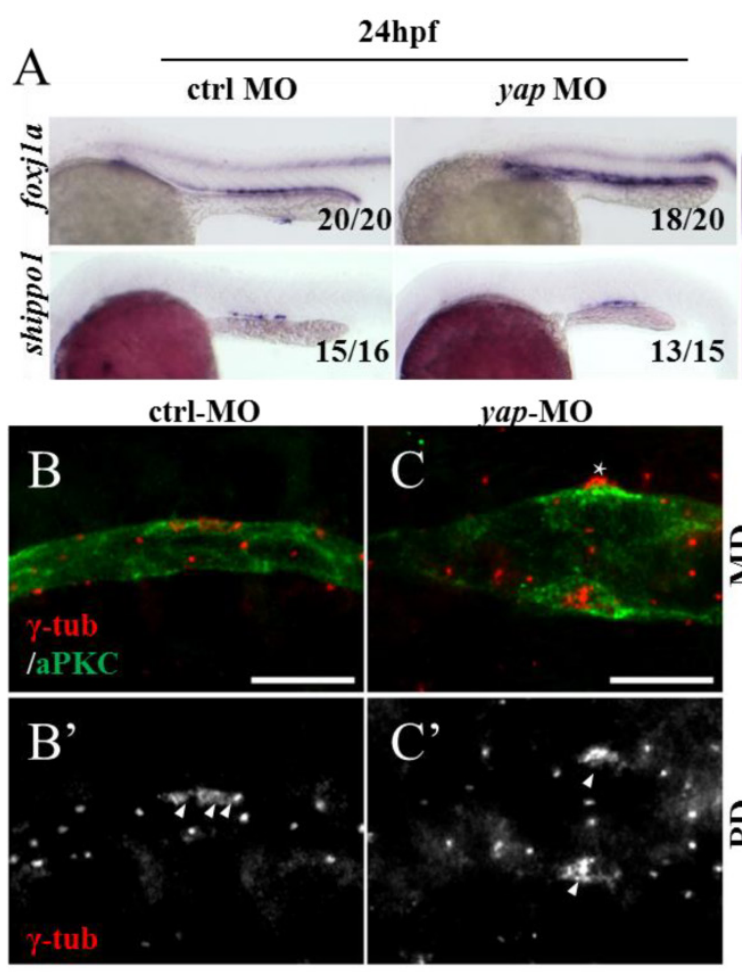

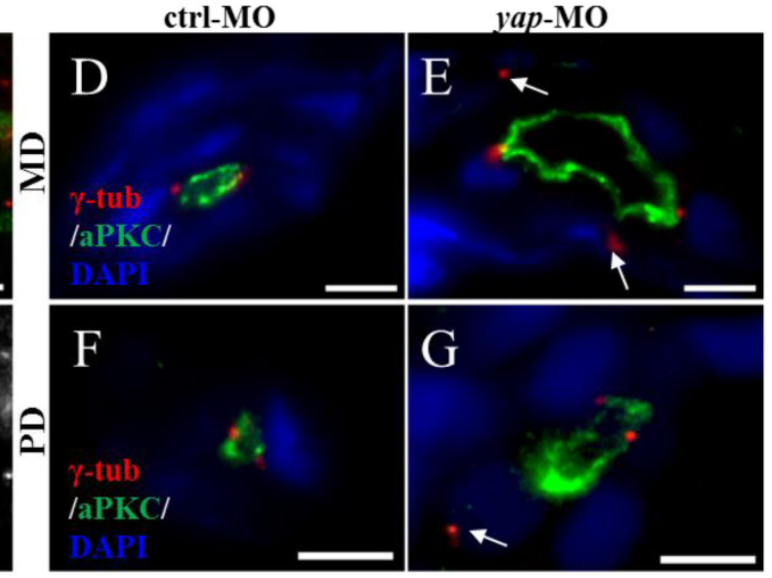

participate in ciliary trafficking pathway. The pattern of defective cilia in yap morphants is very similar to that of IFT complex B mutants and arl13b mutants [9, 15]. IFT complex $B$ is well known for carrying precursors needed for cilia assembly from the synthesis site in the cell body to the assembly site in the cilium. In mouse, IFT20 is the only complex B protein localized to the Golgi apparatus in addition to the cilia and centrosome, and IFT88 is the core protein that assembles other IFT B particles into a complex[44]. Mutation in human ARL13B is associated with Joubert syndrome, and it is also responsible for cilia formation and endocytic recycling traffic[15, 45]. Using $\mathrm{MO}$ based assay, we first titrated MO against yap, ift20, ift88 and arl13b to their suboptimal dosages with hardly detectable pronephric cysts (Fig.7A). Then we combined yap $\mathrm{MO}$ with either of ift20, ift 88 and arl13b MO to test whether they act synergistically by assessing the percentage of cyst formation. Surprisingly, we found all the three groups of co-injected embryos showed significantly increased percentage of pronephric cysts, and the most marked synergy occurred between yap and arl13b (Fig.7B-G). This observation suggests that Yap and Arl13b are most likely to participate in overlapping signaling to maintain normal ciliary transport.

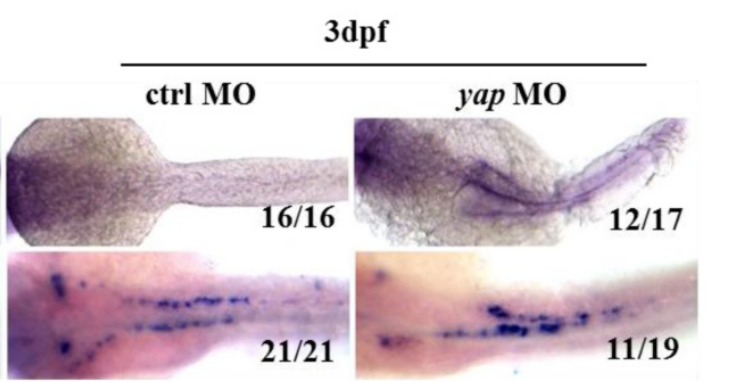

Figure 6. Yap is required for basal body arrangement and apical docking in pronephric duct. (A) The expression level of foxjla (cilia master gene) is increased in 24 h.p.f and 3 d.p.f yap morphant. shippol (a marker of MCCs) expression is unaffected in 24 h.p.f yap morphant, but is accumulated in the medial pronephric duct in 3 d.p.f morphant. 24 h.p.f embryos are lateral views and 3 d.p.f embryos are dorsal views. (B-C') With anti- $\gamma$-tublin staining, basal bodies of MCCs in 3 d.p.f embryos are showed. In control, basal bodies are in a thread-like array at the apical surface (B'; arrowheads), but in morphant, basal bodies are gathered into clusters (C'; arrowheads) and even out of the edge of apical membrane (C; asterisk). Bar: $10 \mu \mathrm{m}$. (D-G) Cross sections staining show that basal bodies are unable to migrate to the apical surface in enlarged pronephric duct of both proximal and distal segments (E and G; arrows). Bar: $5 \mu \mathrm{m}$. $\gamma$-tub, anti- $\gamma$-tublin. 

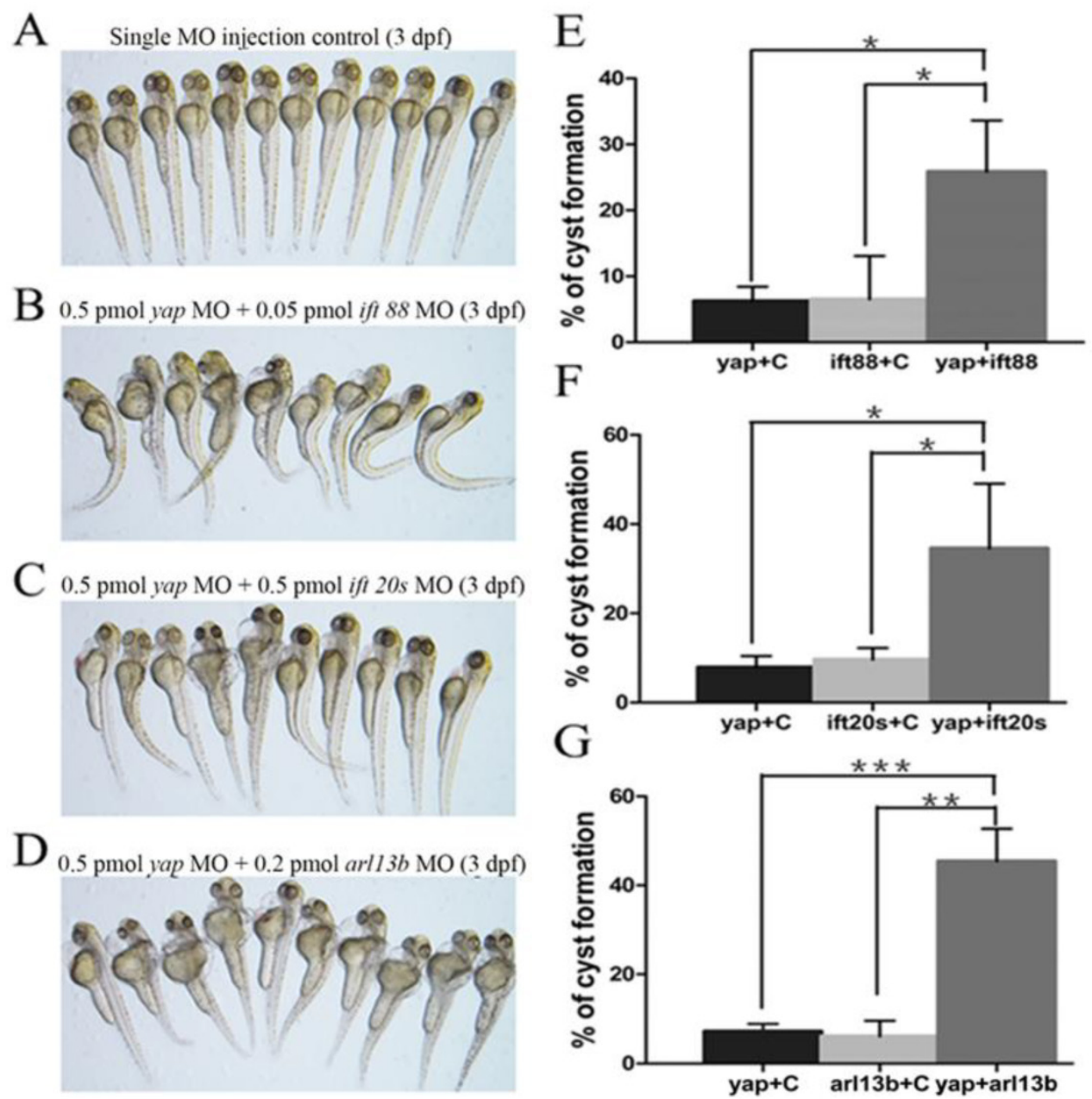

Figure 7. yap genetically interacts with ift20, ift88 and arl / 3b. (A-D) Phenotype of embryos injected with suboptimal yap MO ( $0.5 \mathrm{pmol})$ or/and ift20s $(0.5 \mathrm{pmol})$, ift88 ( $0.05 \mathrm{pmol})$, arl $3 b \mathrm{MO}(0.2 \mathrm{pmol})$ at 3 d.p.f. The single $\mathrm{MO}$ injection control embryos are almost normal (A) while co-injection embryos exhibit dramatically increased percentage of pronephric cysts, heart edema, tail curvature and smaller eyes (B-D). (E-G) Graphs represent the percentage of embryos that develop pronephric cysts. The single MO injection control is treated with suboptimal single $\mathrm{MO}$ and control MO to exclude the effect of mechanical injury to embryos. Each experiment is repeated three times with at least 50 embryos.*: $P<0.05$;**: $P<0.01$;***: $P<0.001$.

\section{Discussion}

The Hippo pathway is a novel signaling and "hot spot" for intensive studies. It is involved in multiple processes such as growth-suppression, cell fate and differentiation, mechanical stress transduction, embryogenesis, and can crosstalk with several important signaling pathways, including Wnt pathway, PCP pathway, Notch Pathway and sonic hedgehog (shh) pathway [20, 24, 29, 46, 47]. Yap is one of transcriptional co-activators of the Hippo pathway, and its relevance to kidney development and PKD has just started to be realized. However, Yap knockout mice is embryonic lethal that limits further study of its in vivo function. In this study, we used zebrafish as a model to investigate the role of Yap in kidney development, ciliogenesis and cystogenesis.

\section{The role of Yap in zebrafish pronephros de- velopment}

Previous study showed that the distribution of Yap is spatially and temporally regulated during mouse kidney development, and knockout of Yap in the cap mesenchymal progenitor cells specifically lead to abnormal nephrogenesis[21]. We found zebrafish Yap is persistently localized in the cytoplasm and apical surface of pronephric duct epithelium cells during embryogenesis, functioning by a non-activated form. Embryos deficient in Yap display discontinuous and dilated pronephric duct, inhibited cell migration as well as defective cloaca, which do not stem from cell fate transition or disruption of cell proliferation, but develop during morphogenesis. The phenotype of pronephric tubule discontinuities and lack of fusion at cloaca is extremely identical to that of cldh17 MO embryos[48]. In addition, Yap was showed to be linked to Fat-cadherin in flies and zebrafish[29]. The cad- 
herin super family is calcium-dependent cell adhesion molecules that play an active role in tissue morphogenesis and patterning. During zebrafish pronephros morphogenesis, pronephric cells have to maintain cell adhesion while migrate collectively[37]. Thus we are in favor of a hypothesis that Yap functions together with cadherin members to affect cell adhesion during morphogenesis of pronephros in zebrafish, and this hypothesis needs to be proved further.

\section{The role of Yap in ciliogenesis}

Multiple evidences support an intricate relationship between the cilium and the Hippo pathway. A list of ciliary proteins as NPHP4 and NPHP9 can regulate the activity of the Hippo pathway directly[49, 50]. In kidneys of Taz knockout mice, cystic lining cells carry fewer and shorter cilia with decreased expression of $\mathrm{Tg} 737$, Kif3a, Dctn5, which encode proteins involved in cilia structure and function[31]. More recently, in vitro study has found that upstream regulator Mst1/2-SAV1complex establish ciliary assembly and ciliary proteins polarized location in a kinase activity-dependent manner. However, loss of Yap using siRNA does not cause ciliary impairment[51].

We show here that yap knockdown in zebrafish with MO assay phenocopies many aspects of ciliopathies, including pronephric cysts, pericardial edema, hydrocephalus, smaller eyes, curved body axis, and these phenotypes are almost in tissues in which yap is highly expressed by ISH. The PKD phenotype and cilia defects could be rescued with full-length yap mRNA and $\triangle$ TAD yap mRNA, However, yap ${ }^{5127 A}$ mRNA does not have the similar rescue effect, suggesting that the role of Yap in ciliogenesis is also in a phosphorylation-dependent manner. We also observe in yap MO embryos, the photoreceptor cell cilia, especially the outer segments, are nearly absent, while in pronephric duct, cilia bundling are disrupted with fewer and shorter single cilia. Without the "fluid-pump" effect of cilia bundles, the pronephric fluid flow can't be generated normally, which in turn prevents the cell migration and the proximal segments convolution. We further demonstrated basal bodies defects in organization and apical membrane docking, and ciliary trafficking genes ift20, ift88 arl13b genetically interact with yap in pronephric cyst formation, suggesting that Yap may participate in multiple ciliary signaling pathways. Together, these results provide a novel insight into the relationship between Yap and ciliogenesis in vivo and further our understanding of the Hippo pathway in the cilium.

\section{The role of Yap in PKD}

The predominant pathological and physiological processes of PKD include excessive proliferation and poor differentiation of the renal epithelium cells, as well as alteration of cell polarity and fluid accumulation in cyst. In cystic and dilated tubules of $P k d 1$ knockout mouse models, autosomal dominant PKD and autosomal recessive PKD patients, Yap nuclear localization is observed in epithelium cells, suggesting activation of Yap may be a common feature in progression of $\mathrm{PKD}[34]$. A simple interpretation is that activated Yap can promote cell proliferation and cyst formation. In this study, we find that the phosphorylated Yap is necessary for $\mathrm{Na}^{+} / \mathrm{K}^{+}$ATPase baso-lateral localization and ciliogenesis in renal tubules of zebrafish, uncovering the essential role of non-activated Yap in maintenance of normal kidney morphology. Activity alteration of Yap may accelerate the progression of PKD as well. One hypothesis arising from our data is that activity inhibition of Yap may delay the progression of PKD and provide a novel therapeutic target.

\section{The PCP pathway and Yap in the kidney}

Planar cell polarity (PCP) refers to coordinated polarization of cells or cell structures within a plane in relation to the tissue axes, and it is visible in directed cell movement during gastrulating embryos or developing kidney and cilia formation in different organisms[52-55]. In Drosophila, the PCP proteins (Fat, Dachsous) are major upstream regulators of $\mathrm{Hpo}$ signaling activity[56]. In addition, multiple lines of evidences support a close relationship between the PCP pathway and Yap in the kidney: In zebrafish, Yap disinhibition participates in pronephric cyst formation after depletion of Fat1 or Scribble, and yap morphants exhibit disrupted dorsoventral expression during gastrulation[25]; In the cystic epithelia of $P k d 1$ depletion mouse, strong nuclear Yap accumulation accompanied with up-regulation of PCP component Four-jointed (Fjx1) was observed[34]. In this study, defects of basal bodies arrangement and polarized docking were found in Yap knockdown embryos, which are also suggestive phenotypes of PCP abnormalities. Given the associations of the PCP pathway with kidney development, PKD and ciliogenesis, we suggest further studies for the potential crosstalk between non-activated Yap and PCP components in the kidney.

\section{Materials and methods}

\section{Zebrafish lines and maintetance}

Wild-type zebrafish (Tubigen and TAB strain) and transgenic $\mathrm{Tg}\left(\mathrm{Na}^{+} / \mathrm{K}^{+}\right.$ATPase:EGFP $)$[42] were raised and maintained as the zebrafish book described [57]. Embryos were kept at $28.5^{\circ} \mathrm{C}$ in E3 solution and 0.003\% PTU (1-phenyl-2-thiourea, Sigma) was added 
to the media at 24 h.p.f to suppress pigmentation.

\section{Plasmid clones}

Zebrafish yap full length cDNA was cloned by PCR into $p C S 2$ vector and a Gateway entry vector. Zebrafish yap5127A cDNA were generated via PCR and subcloning, and this highly conserved phosphorylated site mutation was in the 87th amino acid site in zebrafish actually. mRNA was transcribed using the mMESSAGE mMACHINE SP6 kit (Ambion) as described in the manual after linearization of the constructs with NotI. The NaK ATPase promoter was made using an $8 \mathrm{~kb}$ genomic fragment upsteam of the first fist exon of the $\mathrm{Na}^{+} / \mathrm{K}^{+}$ATPase locus. Using Gateway cloning Kit [36], constructs of a fluorescence reporter system based on Tol2 transposon were generated, in which EGFP and yap/ yap ${ }^{\mathrm{S127A}}$ cDNA were driven by the $\mathrm{Na}^{+} / \mathrm{K}^{+}$ATPase promoter to especially localize in the pronephric ducts ( $p$ Tol2-8kb-EGFP-Yap/ $p$ Tol2-8kb-EGFP-S127A).

\section{Whole-mount in situ hybridization}

Whole-mount in situ hybridization of zebrafish embryos at 6s, 19 s, 24 h.p.f and 72 h.p.f was performed as described previously [58]. The Probes yap, cldh17, nbc1, slc20a1a, trpM7, slc13a1, slc12a1, slc12a3, gata-3, evx1, shippo1, foxj1a have all been reported [20, $41,59]$. The Digoxigenin-labeled antisense riboprobes were made using the mMESSAGE mMACHINE T7 kit (Ambion).

\section{Frozen section and immunohistochemistry}

The embryos were fixed in Dent's fixative (80\% methanol:20\%DMSO) at $-20^{\circ} \mathrm{C}$ overnight or $4 \%$ paraformaldehyde (PFA) in PBST $(0.5 \%$ Tween 20$)$ at room temperature (RT) for 2 hours. For preparation of frozen section, the fixed embryos were gradually dehydrated in 10\%, 20\%, 30\% (w/v) sucrose and embedded in O.C.T in a dry ice bath. Frozen blocks were sectioned at $10 \mu \mathrm{m}$ thickness with a CM1950 cryotome (Leica). Embryos or section were blocked in 10\% normal goat serum and incubated in primary antibody in blocking buffer 2 hours at RT or overnight at $4^{\circ} \mathrm{C}$. After washing 3 times with PBST, 1:500 diluted secondary antibodies were used at RT for 2 hours. Washed embryos were finally embedded and photographed on a Nikon eclipse 80i fluorescence microscope.

\section{Microinjection}

All MOs (Gene Tools) were diluted with RNA only water and injected into embryos at one-cell stage. The sequences of the MOs were described previously: yap5'UTR-MO (5'-CTCTTCTTTCTATCCAACAGA AACC-3' $[20]), \quad$ tp53MO (5'-GCGCCATTGCTTTG CAAGAATTG-3'[35]), ift20SPMO (5'- CAACAACG
TACCTTCATTTTTTCAG-3' [60]), ift885'UTR-MO(5'CTGGGACAAGATGCACATTCTCCAT-3' [61]), arl13b5'UTR-MO (5'-TTTCCCCCCTAAATGCTT TCACTGG-3'[15]). A standard ctrl MO (5'-CСТCTTACCTCAGTTACAATTTATA-3') was used as a negative control. For rescue experiment, 100 pg zebrafish yap full-length mRNA or yap5127A mRNA was co-injected with yap5'UTR-MO into one-two cell stage embyros. $\mathrm{Na}^{+} / \mathrm{K}^{+}$ATPase promoter constructs in the $p$ Tol2 transposase vector $(50 \mathrm{ng} / \mathrm{nl})$ were injected into embryos before one cell stage together with Tol 2 transposase mRNA (50 ng/nl) and imaged at later stages.

\section{Western blot analysis}

Zebrafish embryo lysates were prepared with 1 d.p.f embryos. To remove the yolk protein, the sample was washed with deyolking buffer and washing buffer contain protease inhibitor cocktail (Sigma-Aldrich), and the supernatant was removed following a gentle centrifugation at $300 \mathrm{~g}$ for 3 minutes at $4{ }^{\circ} \mathrm{C}$. The embryos were added with $2 \times$ SDS loading buffer and homogenized completely, and then boiled for 5 minutes following centrifugation at $14000 \mathrm{~g}$ for 10 minutes. Immunoblotting was performed using standard protocols.

\section{Antibodies}

The antibodies used in for IF (immunofluorescence) are: mouse anti alpha $6 \mathrm{~F}\left(\mathrm{Na}^{+} / \mathrm{K}^{+}\right.$ATPase $)$ (1:100,Developmental Studies Hybridoma Bank), rabbit anti PKC $(1: 200$,Santa Cruz), mouse anti acetylated a-tubulin 6-11b-1 (1:2000, Sigma-Aldrich), mouse anti $\gamma$-tubulin (1:100, Sigma-Aldrich), mouse anti GFP (1:500,Roche), rabbit anti YAP (1:50,cell signaling), mouse anti PCNA (1:3000, Sigma-Aldrich), Rabbit antibody to Cldh17 was raised against two different amino acid peptides (QDVNDNYPKLQKT and CPFTFAISRKSQNFEIKP)(1:300,Biomed). The antibodies used in for Western blotting (WB) are: mouse anti Actin (1:3000, Sigma-Aldrich), rabbit anti YAP (1:200, cell signaling). Secondary antibodies used for IF (Alexa Fluor 488 or 594) and WB (horseradish peroxidase-conjugated) are from Invitrogen and ABGENT, respectively.

\section{Statistical Analysis}

Graphpad prism 5.0 was used to derive standard deviation and unpaired Student's $t$-tests.

\section{Supplementary Material}

Supplementary Figures S1-S4.

http://www.ijbs.com/v11p0935s1.pdf 


\section{Abbreviation}

Yap: yes kinase-associated protein; MO: morpholino; PKD: polycystic kidney disease; IFT: intraflagellar transport; Arl13b: ADP-ribosylation factor-like 13B; Taz: transcriptional coactivator with PDZ binding motif; GPCRs: G-protein coupled receptors; ISH: in situ hybridization; LPM: lateral plate mesoderm; IM: intermediate mesoderm; d.p.f: days post fertilization; h.p.f: hours post fertilization; aPKC: anti-atypical PKC; UTR: untranslated region; MCCs: multiciliated cells; SCCs: single ciliated cells

\section{Acknowledgement}

We are grateful for stimulating discussions with members in Cao's lab, Fan Zhang, Miaomiao Jin for providing technical assistance. We would like to thank Dr. Wang Zheng in Prof. Xingzhen Chen's lab for his advice on written English.

\section{Competing Interests}

The authors have declared that no competing interest exists.

\section{References}

[1]. Sharma N, Berbari NF, Yoder BK. Chapter 13 Ciliary Dysfunction in Developmental Abnormalities and Diseases. In: Bradley KY, editor. Current Topics in Developmental Biology. Academic Press; 2008: 371-427.

[2]. Badano JL, Mitsuma N, Beales PL, Katsanis N. The Ciliopathies: An Emerging Class of Human Genetic Disorders. Annual Review of Genomics and Human Genetics. 2006; 7: 125-48.

[3]. Davis EE, Katsanis N. The ciliopathies: a transitional model into systems biology of human genetic disease. Current Opinion in Genetics \& Development. 2012; 22: 290-303.

[4]. Gherman A, Davis EE, Katsanis N. The ciliary proteome database: an integrated community resource for the genetic and functional dissection of cilia. Nat Genet. 2006; 38: 961-2.

[5]. Calvet JP, Grantham JJ. The genetics and physiology of polycystic kidney disease. Semin Nephrol. 2001; 21: 107-23.

[6]. Pazour GJ, San Agustin JT, Follit JA, Rosenbaum JL, Witman GB. Polycystin-2 localizes to kidney cilia and the ciliary level is elevated in orpk mice with polycystic kidney disease. Curr Biol. 2002; 12: R378-80.

[7]. Yoder BK, Hou X, Guay-Woodford LM. The polycystic kidney disease proteins, polycystin-1, polycystin-2, polaris, and cystin, are co-localized in renal cilia. J Am Soc Nephrol. 2002; 13: 2508-16.

[8]. Sun Z, Amsterdam A, Pazour GJ, Cole DG, Miller MS, Hopkins N. A genetic screen in zebrafish identifies cilia genes as a principal cause of cystic kidney. Development. 2004; 131: 4085-93.

[9]. Cao Y, Park A, Sun Z. Intraflagellar transport proteins are essential for cilia formation and for planar cell polarity. J Am Soc Nephrol. 2010; 21: 1326-33.

[10]. Nakanishi K, Sweeney WE, Jr., Avner ED, Murcia NS. Expression of the orpk disease gene during kidney development and maturation. Pediatr Nephrol. 2001; 16: 219-26.

[11]. Jonassen JA, SanAgustin J, Baker SP, Pazour GJ. Disruption of IFT complex A causes cystic kidneys without mitotic spindle misorientation. J Am Soc Nephrol. 2012; 23: 641-51.

[12]. Davenport JR, Watts AJ, Roper VC, Croyle MJ, van Groen T, Wyss JM, et al. Disruption of intraflagellar transport in adult mice leads to obesity and slow-onset cystic kidney disease. Curr Biol. 2007; 17: 1586-94.

[13]. Jonassen JA, San Agustin J, Follit JA, Pazour GJ. Deletion of IFT20 in the mouse kidney causes misorientation of the mitotic spindle and cystic kidney disease. J Cell Biol. 2008; 183: 377-84.

[14]. Garcia-Gonzalo FR, Corbit KC, Sirerol-Piquer MS, Ramaswami G, Otto EA, Noriega TR, et al. A transition zone complex regulates mammalian ciliogenesis and ciliary membrane composition. Nat Genet. 2011; 43: 776-84.

[15]. Duldulao NA, Lee S, Sun Z. Cilia localization is essential for in vivo functions of the Joubert syndrome protein Arl13b/Scorpion. Development. 2009; 136: 4033-42.

[16]. Cantagrel V, Silhavy JL, Bielas SL, Swistun D, Marsh SE, Bertrand JY, et al. Mutations in the cilia gene ARL13B lead to the classical form of Joubert syndrome. Am J Hum Genet. 2008; 83: 170-9.
[17]. Slanchev K, Putz M, Schmitt A, Kramer-Zucker A, Walz G. Nephrocystin-4 is required for pronephric duct-dependent cloaca formation in zebrafish. Hum Mol Genet. 2011; 20: 3119-28.

[18]. Kim YH, Epting D, Slanchev K, Engel C, Walz G, Kramer-Zucker A. A complex of BBS1 and NPHP7 is required for cilia motility in zebrafish. PLoS One. 2013; 8: e72549.

[19]. Williams CL, Li C, Kida K, Inglis PN, Mohan S, Semenec L, et al. MKS and NPHP modules cooperate to establish basal body/transition zone membrane associations and ciliary gate function during ciliogenesis. J Cell Biol. 2011; 192: 1023-41.

[20]. Jiang Q, Liu D, Gong Y, Wang Y, Sun S, Gui Y, et al. yap is required for the development of brain, eyes, and neural crest in zebrafish. Biochem Biophys Res Commun; 2009;: 114-9.

[21]. Reginensi A, Scott RP, Gregorieff A, Bagherie-Lachidan M, Chung C, Lim DS, et al. Yap- and Cdc42-dependent nephrogenesis and morphogenesis during mouse kidney development. PLoS Genet. 2013; 9: e1003380.

[22]. Barron DA, Kagey JD. The role of the Hippo pathway in human disease and tumorigenesis. Clin Transl Med. 2014; 3: 25.

[23]. Varelas X. The Hippo pathway effectors TAZ and YAP in development, homeostasis and disease. Development. 2014; 141: 1614-26.

[24]. Hao J, Zhang Y, Wang Y, Ye R, Qiu J, Zhao Z, et al. Role of extracellular matrix and YAP/TAZ in cell fate determination. Cell Signal. 2014; 26: 186-91.

[25]. Hu J, Sun S, Jiang Q, Sun S, Wang W, Gui Y, et al. Yes-associated protein (yap) is required for early embryonic development in zebrafish (danio rerio). Int J Biol Sci. 2013; 9: 267-78.

[26]. Yin M, Zhang L. Hippo signaling: a hub of growth control, tumor suppression and pluripotency maintenance. J Genet Genomics. 2011; 38: 471-81.

[27]. Fan R, Kim NG, Gumbiner BM. Regulation of Hippo pathway by mitogenic growth factors via phosphoinositide 3-kinase and phosphoinositide-dependent kinase-1. Proc Natl Acad Sci U S A. 2013; 110: 2569-74.

[28]. Genevet A, Tapon N. The Hippo pathway and apico-basal cell polarity. Biochem J. 2011; 436: 213-24

[29]. Skouloudaki K, Puetz M, Simons M, Courbard JR, Boehlke C, Hartleben B, et al. Scribble participates in Hippo signaling and is required for normal zebrafish pronephros development. Proc Natl Acad Sci U S A. 2009; 106: 8579-84.

[30]. Curto M, Cole BK, Lallemand D, Liu CH, McClatchey AI. Contact-dependent inhibition of EGFR signaling by Nf2/Merlin. J Cell Biol. 2007; 177: 893-903.

[31]. Hossain Z, Ali SM, Ko HL, Xu J, Ng CP, Guo K, et al. Glomerulocystic kidney disease in mice with a targeted inactivation of Wwtr1. Proc Natl Acad Sci U S A. $2007 ; 104: 1631-6$.

[32]. Tian Y, Kolb R, Hong JH, Carroll J, Li D, You J, et al. TAZ promotes PC2 degradation through a SCFbeta-Trcp E3 ligase complex. Mol Cell Biol. 2007; 27: 6383-95.

[33]. Makita R, Uchijima Y, Nishiyama K, Amano T, Chen Q, Takeuchi T, et al. Multiple renal cysts, urinary concentration defects, and pulmonary emphysematous changes in mice lacking TAZ. Am J Physiol Renal Physiol. 2008; 294 : F542-53.

[34]. Happe H, van der Wal AM, Leonhard WN, Kunnen SJ, Breuning MH, de Heer E, et al. Altered Hippo signalling in polycystic kidney disease. J Pathol. 2011; 224: $133-42$.

[35]. Gerety SS, Wilkinson DG. Morpholino artifacts provide pitfalls and reveal a novel role for pro-apoptotic genes in hindbrain boundary development. Dev Biol. 2011; 350: 279-89.

[36]. Kwan KM, Fujimoto E, Grabher C, Mangum BD, Hardy ME, Campbell DS, et al. The Tol2kit: a multisite gateway-based construction kit for Tol2 transposon transgenesis constructs. Dev Dyn. 2007; 236: 3088-99.

[37]. Vasilyev A, Liu Y, Mudumana S, Mangos S, Lam PY, Majumdar A, et al. Collective cell migration drives morphogenesis of the kidney nephron. PLoS Biol. 2009; 7: e9.

[38]. Wilson PD. Apico-basal polarity in polycystic kidney disease epithelia. Biochim Biophys Acta. 2011; 1812: 1239-48.

[39]. Sullivan-Brown J, Schottenfeld J, Okabe N, Hostetter CL, Serluca FC, Thiberge SY, et al. Zebrafish mutations affecting cilia motility share similar cystic phenotypes and suggest a mechanism of cyst formation that differs from pkd2 morphants. Dev Biol. 2008; 314: 261-75.

[40]. Yu X, Ng CP, Habacher H, Roy S. Foxj1 transcription factors are master regulators of the motile ciliogenic program. Nat Genet. 2008; 40: 1445-53.

[41]. Hellman NE, Liu Y, Merkel E, Austin C, Le Corre S, Beier DR, et al. The zebrafish foxj1a transcription factor regulates cilia function in response to injury and epithelial stretch. Proc Natl Acad Sci U S A. 2010; 107: 18499-504.

[42]. Liu Y, Pathak N, Kramer-Zucker A, Drummond IA. Notch signaling controls the differentiation of transporting epithelia and multiciliated cells in the zebrafish pronephros. Development. 2007; 134: 1111-22.

[43]. Dawe HR FH, Gull K. Centriole basal body morphogenesis and migration during cliliogenesis in animal cells. J Cell Sci. 2007; 120: 7-15.

[44]. Follit JA, Xu F, Keady BT, Pazour GJ. Characterization of mouse IFT complex B. Cell Motil Cytoskeleton. 2009; 66: 457-68.

[45]. Barral DC, Garg S, Casalou C, Watts GF, Sandoval JL, Ramalho JS, et al. Arl13b regulates endocytic recycling traffic. Proc Natl Acad Sci U S A. 2012; 109: 21354-9.

[46]. Tariki M, Dhanyamraju PK, Fendrich V, Borggrefe T, Feldmann G, Lauth M. The Yes-associated protein controls the cell density regulation of Hedgehog signaling. Oncogenesis. 2014; 3: e112. 
[47]. Dupont S, Morsut L, Aragona M, Enzo E, Giulitti S, Cordenonsi M, et al. Role of YAP/TAZ in mechanotransduction. Nature. 2011; 474: 179-83.

[48]. Horsfield J RA, Reuter K, LaVallie E, Collins-Racie L, Crosier K, Crosier P. Cadherin-17 is required to maintain pronephric duct integrity during zebrafish development. Mech Dev. 2002; 115: 15-26.

[49]. Habbig S, Bartram MP, Muller RU, Schwarz R, Andriopoulos N, Chen S, et al. NPHP4, a cilia-associated protein, negatively regulates the Hippo pathway. J Cell Biol. 2011; 193: 633-42.

[50]. Habbig S, Bartram MP, Sagmuller JG, Griessmann A, Franke M, Muller RU, et al. The ciliopathy disease protein NPHP9 promotes nuclear delivery and activation of the oncogenic transcriptional regulator TAZ. Hum Mol Genet. 2012; 21: 5528-38

[51]. Kim M, Kim M, Lee MS, Kim CH, Lim DS. The MST1/2-SAV1 complex of the Hippo pathway promotes ciliogenesis. Nat Commun. 2014; 5: 5370.

[52]. McNeill H. Planar cell polarity and the kidney. J Am Soc Nephrol. 2009; 20: 2104-11.

[53]. Romaker D, Puetz M, Teschner S, Donauer J, Geyer M, Gerke P, et al. Increased expression of secreted frizzled-related protein 4 in polycystic kidneys. J Am Soc Nephrol. 2009; 20: 48-56.

[54]. Vervenne HB, Crombez KR, Lambaerts K, Carvalho L, Koppen M, Heisenberg $\mathrm{CP}$, et al. Lpp is involved in Wnt/PCP signaling and acts together with Scrib to mediate convergence and extension movements during zebrafish gastrulation. Dev Biol. 2008; 320: 267-77.

[55]. Cevik S, Sanders AA, Van Wijk E, Boldt K, Clarke L, van Reeuwijk J, et al. Active transport and diffusion barriers restrict Joubert Syndrome-associated ARL13B/ARL-13 to an Inv-like ciliary membrane subdomain. PLoS Genet. 2013; 9: e1003977.

[56]. Reddy BVVG, Irvine KD. The Fat and Warts signaling pathways: new insights into their regulation, mechanism and conservation. Development. 2008; 135: 2827-38.

[57]. Westerfield M. The Zebrafish Book: A Guide for the Laboratory Use of Zebrafish (Danio rerio). Eugene: University of Oregon Press; 2000.

[58]. Jowett T YY. Double fluorescent in situ hybridization to zebrafish embryos. Trends Genet 1996; 12: 387-9.

[59]. Wingert RA, Selleck R, Yu J, Song HD, Chen Z, Song A, et al. The cdx genes and retinoic acid control the positioning and segmentation of the zebrafish pronephros. PLoS Genet. 2007; 3: 1922-38.

[60]. Omori Y, Zhao C, Saras A, Mukhopadhyay S, Kim W, Furukawa T, et al. Elipsa is an early determinant of ciliogenesis that links the IFT particle to membrane-associated small GTPase Rab8. Nat Cell Biol. 2008; 10: 437-44.

[61]. Kramer-Zucker AG, Olale F, Haycraft CI, Yoder BK, Schier AF, Drummond IA. Cilia-driven fluid flow in the zebrafish pronephros, brain and Kupffer's vesicle is required for normal organogenesis. Development. 2005; 132: 1907-21. 